\title{
MicroRNA-20a promotes the proliferation and cell cycle of human osteosarcoma cells by suppressing early growth response 2 expression
}

\author{
WENKUN ZHUO ${ }^{1,2}$, WEIMING GE ${ }^{3}$, GUOLIN MENG ${ }^{1}$, SHUAIJUN JIA ${ }^{4}$, XIANG ZHOU $^{1}$ and JIAN LIU ${ }^{1}$ \\ ${ }^{1}$ Department of Orthopedics and Traumatology, Institute of Orthopedics and Traumatology, Xijing Hospital, \\ The Fourth Military Medical University, Xi'an, Shanxi 710000; ${ }^{2}$ Department of Orthopedics and Traumatology, \\ Jinan Military General Hospital, Jinan, Shandong 250000; ${ }^{3}$ Department of Orthopedics and Traumatology, \\ The PLA Fifth Thirty Four Hospital, Luoyang, Henan 471000; ${ }^{4}$ Department of Orthopedics, \\ Shanxi Hospital of Chinese Armed Police Forces, Xi'an, Shanxi 710000, P.R. China
}

Received September 30, 2014; Accepted June 25, 2015

DOI: $10.3892 / \mathrm{mmr} .2015 .4098$

\begin{abstract}
MicroRNAs (miRNAs) are crucial in cancer development. However, the underlying mechanisms of miRNAs in osteosarcoma (OS) remain largely uncharacterized. The present study investigated the role of miR-20a in OS cell proliferation. It was determined that miR-20a expression is markedly upregulated in OS tissues and cells compared with the matched adjacent normal tissues and h-FOB human osteoblast cell lines. Ectopic expression of miR-20a promoted the proliferation and anchorage-independent growth of OS cells, whereas inhibition of miR-20a reduced this effect. Bioinformatics analysis further revealed early growth response 2 (EGR2), as a potential target of miR-20a. Data from luciferase reporter assays showed that miR-20a directly binds to the 3'-untranslated region (3'-UTR) of EGR 2 mRNA and represses expression at the transcriptional and translational levels. In functional assays, miR-20a promoted OS cell proliferation and the cell cycle, which could be suppressed by an inhibitor of miR-20a. In conclusion, the data provide compelling evidence that miR-20a functions as an onco-miRNA, which is important in promoting cell proliferation in OS, and its oncogenic effect is mediated primarily through direct suppression of EGR2 expression.
\end{abstract}

\section{Introduction}

Osteosarcoma (OS) is a high-grade malignant bone neoplasm, which has become one of the most common primary malignant

Correspondence to: Dr Jian Liu, Department of Orthopedics and Traumatology, Institute of Orthopedics and Traumatology, Xijing Hospital, The Fourth Military Medical University, 169 Changlexi Road, Xi'an, Shanxi 710000, P.R. China

E-mail: jianliuxian@163.com

Key words: microRNA-20a, osteosarcoma, early growth response 2, cell proliferation, cell cycle bone tumors in children and young adults (1). The majority of failures of osteosarcoma treatment are due to resistance to chemotherapy $(2,3)$. Thus, there is an urgent need to elucidate the underlying molecular mechanisms of OS and to determine an appropriate gene therapy strategy in OS treatment (4).

Recent studies have demonstrated that microRNAs (miRNAs), are small non-coding RNAs of 18-25 nt. They have the ability to regulate cellular processes, such as cell proliferation, apoptosis, invasion and differentiation, suggesting that they could be involved in cancer (5-7). Previous studies have found that miR-20a is upregulated in several types of cancer, suggesting that it may be pivotal in tumorigenesis and tumor progression (8-10). In the present study, the biological effects and the potential mechanisms of miR-20a in OS were investigated. The role of miR-20a in OS development was investigated by MTT and cell cycle assays. In addition, the present study explored the regulatory role of miR-20a on EGR2 in OS cells, and investigated its function in cell proliferation.

\section{Materials and methods}

Clinical specimens. Eight human osteosarcoma (OS) tissues and matched adjacent normal tissues (ANT) were obtained from patients with OS (age, 49-63 years; 4 females, 4 males) at the Institute of Orthopedics and Traumatology, Xijing Hospital, The Fourth Military Medical University (Xi'an, China). This study was approved by the ethics committee of Xijing Hospital. Written informed consent was obtained from all patients. Tissue samples were collected during surgery, immediately frozen in liquid nitrogen and stored until total RNA or protein was extracted.

Cell culture. MG-63, Saos-2 and SW1353 human osteosarcoma cell lines and h-FOB human osteoblast cell lines were purchased from the Cell Bank of Chinese Academy of Sciences (Shanghai, China) and grown in Dulbecco's modified Eagle's medium (DMEM, Gibco-BRL, Carlsbad, CA, USA) supplemented with $10 \%$ fetal bovine serum (FBS, Sigma-Aldrich, St. Louis, MO, USA) and $100 \mathrm{U} / \mathrm{ml}$ penicillin-streptomycin 
(Invitrogen Life Technologies, Carlsbad, CA, USA). Cell lines were cultured in a humidified incubator at $37^{\circ} \mathrm{C}$ in an atmosphere of $5 \% \mathrm{CO} 2$ and $95 \%$ air.

Plasmids, small interfering (si)RNA and transfection. For ectopic expression of EGR2, EGR2 open reading frames with 3'-UTRs were amplified using polymerase chain reaction (PCR) and subcloned into pEGFP-N1 using the following primers: Sense: 5'-CCCTCGAGATCCCAGGCTCAGTCCAACC-3 and antisense: 5'-CCAAGCTTAGGTGTCCGGGTCCG AGA-3' (Invitrogen Life Technologies). miR-20a mimic, miR-20a inhibitor and negative control (NC) were purchased from GeneCopoeia (Guangzhou, China) and transfected into OS cells using Lipofectamine ${ }^{\circledR} 2000$ reagent (Invitrogen Life Technologies) according to the manufacturer's instructions.

For EGR2 depletion, EGR2-siRNA (SI04948762) and negative control siRNA were purchased from Qiagen China (Shanghai) Co. Ltd. (Shanghai, China). Transfection of siRNAs was performed using Lipofectamine 2000, according to the manufacturer's instructions.

RNA extraction and reverse transcription-quantitative $(R T-q) P C R$. For miRNA quantification, total RNA including microRNAs was extracted from culture cells and patient samples using the mirVana miRNA Isolation kit (Ambion, Austin, TX, USA) according to the manufacturer's instructions, and then cDNA was synthesized from $5 \mathrm{ng}$ of total RNA using the Taqman ${ }^{\circledR}$ miRNA Reverse Transcription kit (Applied Biosystems, Foster City, CA, USA). The expression levels of miR-20a were quantified using the miRNA-specific TaqMan ${ }^{\circledR}$ MiRNA Assay kit (Applied Biosystems) by the 7500 Sequence Detection system (Applied Biosystems). The relative miR-20a expression levels after normalization to U6 small nuclear RNA were calculated using 2-[(Ct of miR-20a) - (Ct of U6)]. qPCR was performed using an SYBR kit [Qiagen China (Shanghai) Co., Ltd.]. The PCR reaction conditions for all assays were as follows: $95^{\circ} \mathrm{C}$ for $30 \mathrm{sec}$, followed by 40 cycles of amplification $\left(95^{\circ} \mathrm{C}\right.$ for $5 \mathrm{sec}, 59^{\circ} \mathrm{C}$ for $30 \mathrm{sec}$ and $72^{\circ} \mathrm{C}$ for $30 \mathrm{sec})$. The primers which were synthesized by a TaqMan ${ }^{\circledR}$ Gene Expression Assay (Invitrogen Life Technologues) were used as follows: Cyclin D1 (Hs00765553/m1) and p21 (Hs00159357/m1). Expression data were normalized to the geometric mean of GAPDH (Hs02758991/g1) to control the variability in expression levels and calculated as $2-[(\mathrm{Ct}$ of cyclin D1 and p21) - (Ct of GAPDH)].

MTT assays and colony formation. For MTT assays, Saos-2 cells were seeded into 96-well plates in medium containing $10 \% \mathrm{FBS}$ at 3,000 cells/well. In brief, $20 \mu \mathrm{l}$ of $5 \mathrm{mg} / \mathrm{ml}$ MTT solution (Sigma-Aldrich) was added to each well and incubated at $37^{\circ} \mathrm{C}$ for $4 \mathrm{~h}$. After the incubation period, the medium was removed from each well and the resulting MTT formazan was solubilized in $150 \mu \mathrm{l}$ dimethyl sulfoxide (Sigma-Aldrich). The absorbance at $490 \mathrm{~nm}$ was measured in a Thermo Scientific Multiskan (Thermo Fisher Scientific, Rockford, IL, USA).

For the colony formation assay, Saos- 2 cells were plated into three $6-\mathrm{cm}$ cell culture dishes $\left(1 \times 10^{3}\right.$ cells/well) and incubated for 10 days in medium containing $10 \%$ FBS. The colonies were washed with phosphate-buffered saline (PBS) and stained with $1.0 \%$ crystal violet (Beyotime Institute of
Biotechnology, Haimen, China) for $30 \mathrm{sec}$ after fixation with $10 \%$ formaldehyde (Beyotime Institute of Biotechnology) for $15 \mathrm{~min}$. The number of colonies, defined as $>50$ cells/colony were counted under an inverted fluorescent microscope (MoticAE30; Microscope Systems, Ltd., Glasgow, UK). Three independent experiments were performed and the data was calculated using a paired t-test.

Cell cycle assay. Cells were harvested, washed twice with cold PBS, fixed in 70\% ice-cold ethanol, and incubated with propidium iodide (PI) (Beyotime Institute of Biotechnology) and RNase A (Beyotime Institute of Biotechnology), then analyzed by fluorescence-activated cell sorting (FACScan; BD Biosciences, San Jose, CA, USA). Each sample was run in triplicate.

Luciferase assays. To construct a luciferase reporter vector, the EGR2 3'-UTR fragment containing putative binding sites for miR-20a was amplified using PCR and cloned in the psiCHECK-2 vector (Promega Corporation, Madison, WI, USA). These constructs $(1 \mu \mathrm{g})$ were co-transfected with $1 \mu \mathrm{g}$ control or miR-20a precursor into Saos-2 cells. Dual-luciferase activity assays (cat. no. RG027; Beyotime Institute of Biotechnology) were assayed $48 \mathrm{~h}$ post-transfection according to the manufacturer's instructions.

Western blotting. Protein lysates were prepared, the equivalent aliquotsof proteinswere electrophoresed on a 10\% SDS/polyacrylamide gel (Beyotime Institute of Biotechnology) and transferred to nitrocellulose membranes (Invitrogen Life Technologies). The membrane was blocked in Tris-buffered saline-Tween 20 (Institute of Biotechnology) supplemented with 5\% milk (Institute of Biotechnology) for $2 \mathrm{~h}$ at room temperature, and then incubated overnight at $4^{\circ} \mathrm{C}$ with anti-EGR2 (1:1,000; cat. no. ab108399; Abcam, Cambridge, MA, USA), anti-cyclin D1 (1:1,000; cat. no. ab16663; Abcam) and anti-p21 (1:1,000; cat. no. 2947; Cell Signaling Technology, Inc., Beverly, MA, USA). $\alpha$-tubulin (1:500; cat. no. T9026; Sigma-Aldrich) acted as a protein loading control. Following incubation with secondary antibody (horseradish peroxidase-conjugated) for $2 \mathrm{~h}$ at room temperature, immunocomplexes were visualized using the Enhanced Chemiluminesence Advanced Western Blotting Detection kit (GE Healthcare Bio-Sciences, Pittsburgh, PA, USA) according to the manufacturer's instructions.

Statistical analysis. Statistical analysis was performed using SPSS 15.0 (SPSS, Inc., Chicago, IL, USA). Statistical analyses were conducted by Student's t-test. $\mathrm{P}<0.05$ was considered to indicate a statistically significant difference.

\section{Results}

miR-20a expression is upregulated in OS tissues and OS cell lines. To investigate the role of miR-20a in OS development, the expression levels of miR-20a in OS tissues and OS cells were first evaluated by RT-qPCR (Fig. 1A). The results showed that the expression levels of miR-20a were upregulated in the OS tissues compared with in the matched ANTs, and all three tested OS cell lines had significantly upregulated miR-20a levels compared with the h-FOB human osteoblast cell lines 
A

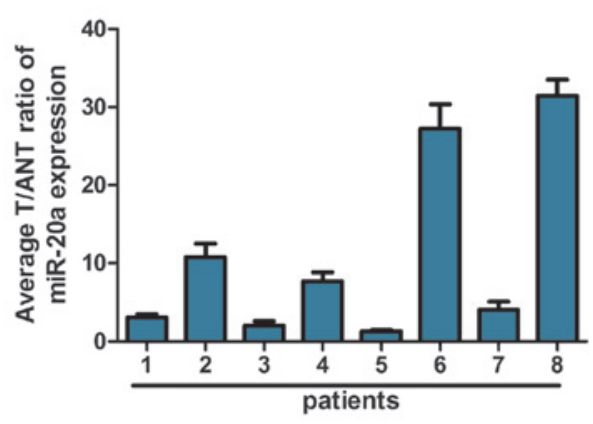

B

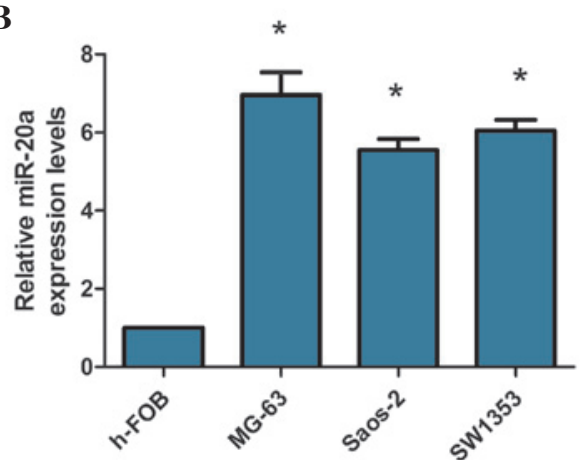

Figure 1. Expression of miR-20a in human OS tissues and cell lines. (A) Relative miR-20a expression levels in 8 paired primary OS tissues and matched ANTs from the same patient were detected by PCR analysis. (B) RT-qPCR analysis of miR-20a expression in h-FOB human osteoblast cell lines and MG-63, Saos-2 and SW1353 OS cell lines. Experiments were repeated at least three times. Each bar represents the mean of three independent experiments. ${ }^{*} \mathrm{P}<0.05$, compared with h-FOB. miR, microRNA; OS, osteosarcoma; T, primary OS tissues; ANT, adjacent normal tissues; RT-qPCR, reverse transcription-quantitative polymerase chain reaction.

A

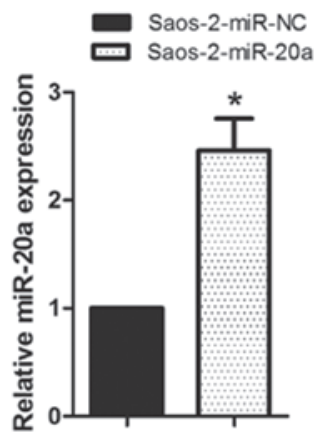

C

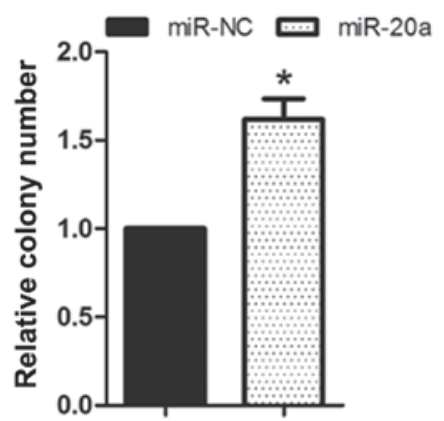

B

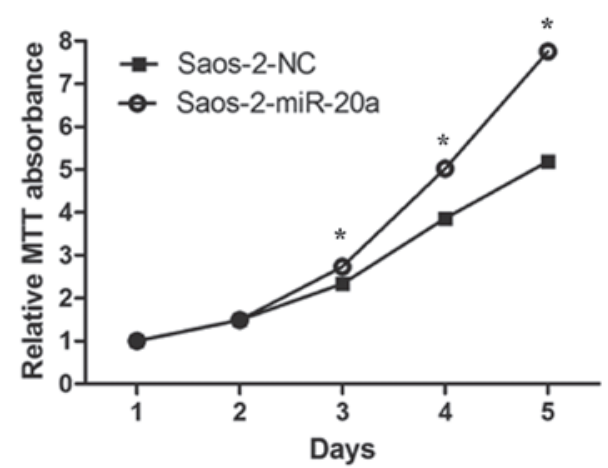

D

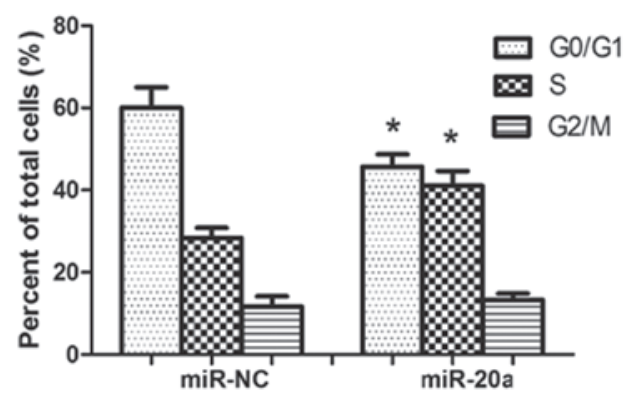

Figure 2. miR-20a upregulation promoted OS cell proliferation. (A) Validation of miR-20a expression levels after transfection by PCR analysis. (B) MTT assays revealed that inhibition of miR-20a promoted growth of the Saos-2 OS cell line. (C) Representative quantification of crystal violet-stained cell colonies. (D) Cell cycle distribution in cells with miR-20a transfection. Each bar represents the mean of three independent experiments. "P<0.05, compared with NC. miR, microRNA; PCR, polymerase chain reaction; OS, osteosarcoma.

(Fig. 1B). These results showed that miR-20a exhibited a crucial role in OS and suggest that miR-20a is significantly increased in OS and may serve as a prognostic marker for patients with OS.

miR-20a promotes $O S$ cell proliferation and cell cycle progression. As miR-20a was significantly upregulated in OS tissues and OS cell lines, it was investigated whether miR-20a promotes cell proliferation and the cell cycle of OS cells (Figs. 2 and 3). Saos-2 cells were transfected with miR-20a mimics, miR-20a inhibitor or the respective controls, both of them showed great transfection efficiency (Figs. 2A and 3A).
The MTT assay showed that the cell growth rate was significantly higher in miR-20a-transduced Saos-2 cells than in miR-NC-transfected cells (Fig. 2B). Colony formation assays consistently showed that enforced expression of miR-20a significantly enhanced the number of Saos- 2 cell colonies after 14 days of culture compared with the controls (Fig. 2C). By contrast, the cell growth rates and colony numbers of Saos-2 cells transfected with miR-20a-in were significantly lower than those transfected with NC (Fig. 3B and C). To determine whether modulating cell viability was a result of the cell cycle, flow cytometry was used. After $48 \mathrm{~h}$ transfection, miR-20a mimics decreased the proportion of Saos- 2 cells 
A

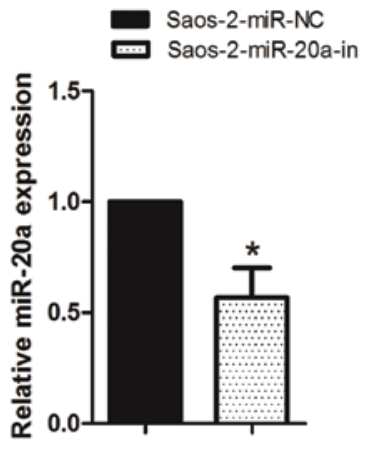

C

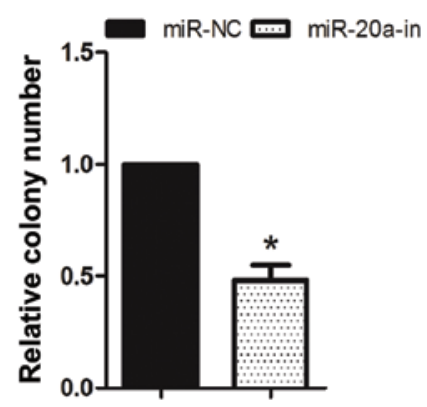

B

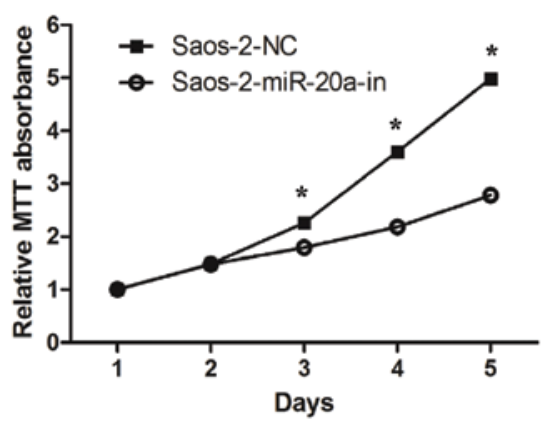

D

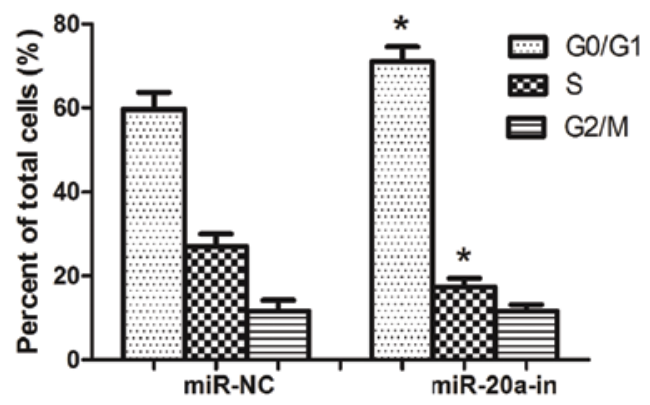

Figure 3. miR-20a downregulation inhibited OS cell proliferation. (A) Validation of miR-20a expression levels after transfection by PCR analysis. (B) MTT assays revealed that downregulation of miR-20a inhibited the growth of Saos-2 OS cells. (C) Representative quantification of crystal violet-stained cell colonies. (D) Cell cycle distribution in cells with miR-20a-in transfection. Each bar represents the mean of three independent experiments. ${ }^{*} \mathrm{P}<0.05$, compared with NC. miR, microRNA; OS, osteosarcoma; PCR, polymerase chain reaction.

A

$$
\begin{aligned}
& \text { EGR2-3'UTR 5'AGCAAAACUGAUGUGGCACUUUA3' } \\
& \text { |1 | |111111 } \\
& \text { miR-20a 3'GAUGGACGUGAUAUU--CGUGAAAUs' } \\
& \text { I I I | | I }
\end{aligned}
$$

B

EGR2

a-Tubulin
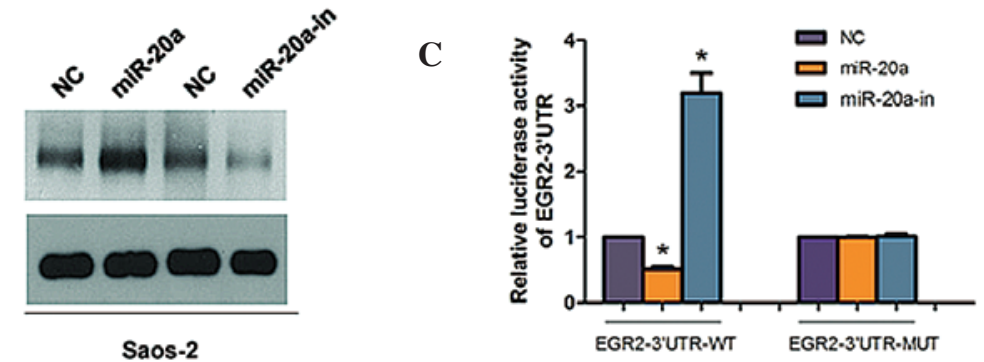

Figure 4. miR-20a suppresses EGR2 expression by directly targeting the EGR2 3'-UTR. (A) Predicted miR-20a target sequence in the 3'-UTR of EGR-2 (EGR-2-3'-UTR) and positions of mutated nucleotides in the 3'-UTR of EGR-2 (EGR-2-3'-UTR-mut). (B) Western blot analysis of EGR-2 expression in cells transfected with miR-20a or the miR-20a inhibitor. $\alpha$-tubulin served as the loading control. (C) Luciferase reporter assay of the indicated cells transfected with the pGL3-EGR-2-3'-UTR reporter and miR-20a or miR-20a-in with oligonucleotides. "P<0.05, compared with NC. miR, microRNA; EGR2, early growth response 2; UTR, untranslated region; mut, mutant.

in the G0/G1-phase and increased the proportion of Saos-2 cells in the S-phase compared with those transfected with $\mathrm{NC}$ (Fig. 2D). However, miR-20a-in increased the proportion of Saos-2 cells in the G0/G1-phase and decreased the proportion of Saos-2 cells in S-phase compared with those transfected with NC (Fig. 3D). These results showed that miR-20a promoted OS cell tumorigenicity in vitro.

miR-20a directly targets EGR2 by binding to its 3'-UTR in OS. It is generally accepted that miRNAs regulate the expression of mRNAs by targeting the mRNA 3'UTR. Bioinformatics analysis revealed that EGR2, is a member of a multi-gene family encoding zinc finger proteins and is crucial in the regulation of cellular proliferation and the cell cycle, as a putative target of miR-20a (11-13). Therefore, EGR2 was selected as a target for further analysis (Fig. 4A).

To determine whether miR-20a affects EGR2 expression, expression of EGR2 was detected in the Saos-2 cells, which were transfected with miR-20a mimics, miR-20a-in or the respective controls. Western blot analysis showed that miR-20a mimics markedly suppressed EGR2 protein levels in Saos-2 cells (Fig. 4B), while miR-20a-in clearly promoted EGR2 protein expression. To verify the effect of miR-20a on the inhibition of EGR2 expression, it was examined whether EGR2 is regulated by miR-20a through direct binding to its 3'UTR. EGR2 3'-UTR (wild or mut type) vector was cotransfected in Saos-2 cells with miR-20a mimic or miR-20a-in and then luciferase activity was measured. As shown in Fig. 4C, 


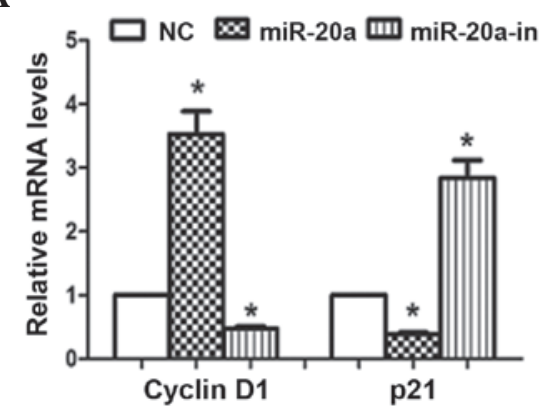

B

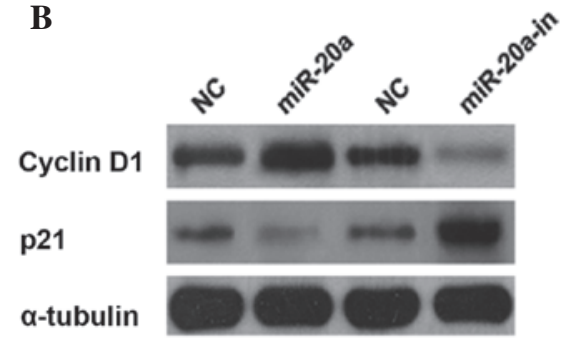

Figure 5. miR-20a altered the levels of proteins associated with cell proliferation and the cell cycle in Saos-2 cells. (A) Reverse transcription-quantitative polymerase chain reaction analysis of expression of cyclin D1 and p21 in Saos-2 cells. (B) Western blot analysis of cyclin D1 and p21 protein in Saos-2 cells. $\alpha$-tubulin served as the loading control. "P $<0.05$, compared with NC. miR, microRNA.

A

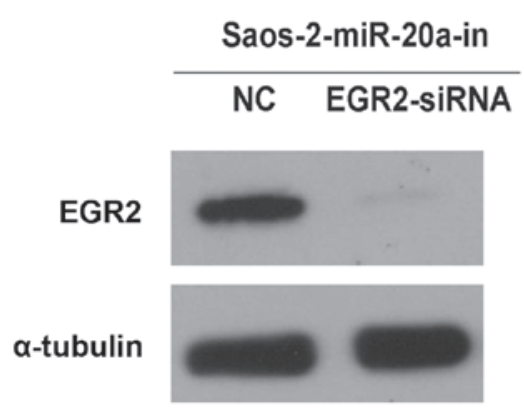

B

Saos-2-miR-20a-in+NC

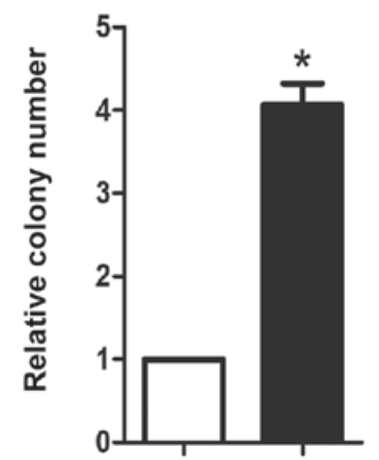

Figure 6. EGR-2 upregulation is required for miR-20a-in-induced proliferation of OS cells. (A) Western blot analysis verified that silencing EGR-2 effectively decreased the expression of EGR-2 in miR-20a-in-transfected Saos-2 cells. (B) miR-20a-in-transfected Saos-2 cells after transfection with EGR-2-siRNA promoted cell colony formation. Each bar represents the mean of three independent experiments. "P<0.05, compared with NC. EGR2, early growth response 2; miR, microRNA; OS, osteosarcoma; siRNA, small interfering RNA.

significant repression of luciferase activity was observed in Saos-2 cells cotransfected with pGL-3-EGR2 3'-UTR vector and miR-20a mimic, whereas miR-20a-in increased luciferase activity. These results, demonstrated that EGR2 is a target of miR-20a.

miR-20a alters the levels of proteins associated with proliferation and the cell cycle in OS cells. To investigate the mechanism underlying cell proliferation and the cell cycle, the effect of miR-20a on critical cell-proliferation and cell cycle related regulators cyclin D1 and p21 were analyzed. The cyclin-dependent kinase inhibitor p21 and cyclin D1 are two miR-20a targeted proteins that negatively (p21) and positively (cyclin D1) regulate control cell cycle progression and proliferation $(14,15)$. As shown in Fig. 5, cyclin D1 mRNA and protein were upregulated in Saos-2 cells transfected with miR-20a mimic, but the levels were decreased in the cells transfected with miR-20a-in, relative to control cells. In addition, overexpression of miR-20a caused the downregulation of p21 (mRNA and protein), but upregulation in the cells tranfected with miR-20a-in. Thus providing further evidence that miR-20a is important in OS cell proliferation and the cell cycle.

EGR2 upregulation is required for miR-20a-in-induced proliferation of OS cells. To further investigate the role of
EGR2 promotion in miR-20a-in-induced OS proliferation, the effects of EGR2 downregulation on OS cell proliferation were first examined. As predicted, western blot analysis verified that EGR2-siRNA effectively decreased the expression of EGR2 in miR-20a-in-transfected Saos-2 cells (Fig. 6A). Colony formation assays showed that EGR2-silencing and miR-20a-in-transfection in Saos-2 cells exhibited an additive effect on cell proliferation (Fig. 6B). These results demonstrate that direct EGR2 downregulation is required for miR-20a-induced OS cell proliferation.

\section{Discussion}

Growing evidence suggests that microRNAs exhibit crucial roles in development and in the progression of human cancers, such as OS (16-20). miRNAs are a large family of gene regulators that negatively regulate their target mRNAs in a sequence-specific manner $(6,21)$. The effects of miR-20a have been described in numerous types of cancer cells $(8,10)$. However, it was uncertain whether dysregulation of miR-20a was associated with the progression of OS. In the present study, miR-20a expression was examined in human OS tissues, the matched adjacent normal tissues, OS cell lines (MG-63, Saos-2, and SW1353) and h-FOB human osteoblast cell lines. It was demonstrated that miR-20a expression was 
markedly upregulated in OS tissues and OS cells, suggesting that miR-20a is important in malignant processes in OS.

Bioinformatic analysis revealed that EGR2, a novel member of the Egr family of genes encoding immediate-early transcription factors, is a target gene of miR-20a. Furthermore, direct binding of miR-20a to the 3'-UTR of EGR2 mRNA was observed and it was demonstrated that miR-20a overexpression suppresses EGR2 expression in OS cells, while inhibition of miR-20a increases its expression.

Given that EGR2 is key in the regulation of cell proliferation and the cell cycle $(12,22)$ the effect of miR-20a on these processes in OS cells was investigated. Saos- 2 cells were transfected with miR-20a or miR-20a-in to upregulate or downregulate miR-20a expression. It was demonstrated that ectopic expression of miR-20a enhanced the cell proliferation of Saos-2 cells. Conversely, inhibition of miR-20a inhibited cell proliferation. Using flow cytometric analysis, it was observed that miR-20a-transfected Saos-2 cells entered the $\mathrm{S}$ phase earlier and inhibition of miR-20a arrested cell cycle progression at the G0/G1 boundary. More specifically, to determine the potential underlying mechanism, the expression of cell proliferation and cycle-associated molecules cyclin D1 and p21 was investigated, the results showed that the molecular mechanism by which miR-20a promotes OS cancer cell proliferation was due to acceleration of the G1/S phase transition, upregulation of cyclin D1 and downregulation of p21 in cells overexpressing miR-20a (23-25). Furthermore, EGR2-silencing in miR-20a-in-transfected Saos-2 cells exhibited a positive effect to promote cell proliferation, suggesting that direct EGR2 downregulation is required for miR-20a-induced OS cell proliferation.

In conclusion, the current study revealed that upregulation of miR-20a is a common event underlying OS. Furthermore, miR-20a functions as an oncogene by directly targeting EGR2. These findings collectively suggest that miR-20a may serve as a promising prognostic and therapeutic target for OS.

\section{Acknowledgements}

This study was supported by the Institute of Orthopedics and Traumatology, Xijing Hospital.

\section{References}

1. Ottaviani G and Jaffe N: The epidemiology of osteosarcoma. Cancer Treat Res 152: 3-13, 2009.

2. Ahmed AA, Zia H and Wagner L: Therapy resistance mechanisms in Ewing's sarcoma family tumors. Cancer Chemother Pharmacol 73: 657-663, 2014.

3. Constantinidou A, Pollack S, Loggers E, Rodler E and Jones RL: The evolution of systemic therapy in sarcoma. Expert Rev Anticancer Ther 13: 211-223, 2013.

4. Miao J, Wu S, Peng Z, Tania M and Zhang C: MicroRNAs in osteosarcoma: Diagnostic and therapeutic aspects. Tumour Biol 34: 2093-2098, 2013.
5. Esquela-Kerscher A and Slack FJ: Oncomirs - microRNAs with a role in cancer. Nat Rev Cancer 6: 259-269, 2006.

6. Calin GA and Croce CM: MicroRNA signatures in human cancers. Nat Rev Cancer 6: 857-866, 2006.

7. Sun K and Lai EC: Adult-specific functions of animal microRNAs. Nat Rev Genet 14: 535-548, 2013.

8. Xiong Y, Zhang L and Kebebew E: MiR-20a is upregulated in anaplastic thyroid cancer and targets LIMK1. PLoS One 9: e96103, 2014

9. Zhao S, Yao D, Chen J and Ding N: Circulating miRNA-20a and miRNA-203 for screening lymph node metastasis in early stage cervical cancer. Genet Test Mol Biomarkers 17: 631-636, 2013.

10. Chang Y, Liu C, Yang J, Liu G, Feng F, Tang J, Hu L, Li L, Jiang F, Chen C, et al: MiR-20a triggers metastasis of gallbladder carcinoma. J Hepatol 59: 518-527, 2013.

11. Joseph LJ, Le Beau MM, Jamieson GA Jr, Acharya S, Shows TB, Rowley JD and Sukhatme VP: Molecular cloning, sequencing, and mapping of EGR2, a human early growth response gene encoding a protein with "zinc-binding finger" structure. Proc Natl Acad Sci USA 85: 7164-7168, 1988.

12. Li X,Zhang Z, Yu M, Li L, Du G, Xiao W and Yang H: Involvement of miR-20a in promoting gastric cancer progression by targeting early growth response 2 (EGR2). Int J Mol Sci 14: 16226-16239, 2013.

13. Sumitomo S, Fujio K, Okamura T and Yamamoto K: Egr2 and Egr3 are the unique regulators for systemic autoimmunity. JAK-STAT 2: e23952, 2013.

14. Yu Z, Wang C, Wang M, Li Z, Casimiro MC, Liu M, Wu K, Whittle J, Ju X, Hyslop T, et al: A cyclin D1/microRNA 17/20 regulatory feedback loop in control of breast cancer cell proliferation. J Cell Biol 182: 509-517, 2008.

15. Wang Z, Liu M, Zhu H, Zhang W, He S, Hu C, Quan L, Bai J and $\mathrm{Xu}$ N: Suppression of $\mathrm{p} 21$ by c-Myc through members of miR-17 family at the post-transcriptional level. Int J Oncol 37: 1315-1321, 2010.

16. Arabi L, Gsponer JR, Smida J, Nathrath M, Perrina V, Jundt G, Ruiz C, Quagliata L and Baumhoer D: Upregulation of the miR-17-92 cluster and its two paraloga in osteosarcoma - reasons and consequences. Genes Cancer 5: 56-63, 2014.

17. Tian Q, Jia J, Ling S, Liu Y, Yang S and Shao Z: A causal role for circulating miR-34b in osteosarcoma. Eur J Surg Oncol 40: 67-72, 2014.

18. Xu JQ, Liu P, Si MJ and Ding XY: MicroRNA-126 inhibits osteosarcoma cells proliferation by targeting Sirt1. Tumour Biol 34: 3871-3877, 2013.

19. Cao ZQ, Shen Z and Huang WY: MicroRNA-802 promotes osteosarcoma cell proliferation by targeting p27. Asian Pac J Cancer Prev 14: 7081-7084, 2013.

20. Tang M, Lin L, Cai H, Tang J and Zhou Z: MicroRNA-145 downregulation associates with advanced tumor progression and poor prognosis in patients suffering osteosarcoma. Onco Targets Ther 6: 833-838, 2013

21. van Kouwenhove M, Kedde M and Agami R: MicroRNA regulation by RNA-binding proteins and its implications for cancer. Nat Rev Cancer 11: 644-656, 2011.

22. Unoki M and Nakamura Y: Growth-suppressive effects of BPOZ and EGR2, two genes involved in the PTEN signaling pathway. Oncogene 20: 4457-4465, 2001.

23. Baldin V, Lukas J, Marcote MJ, Pagano M and Draetta G: Cyclin D1 is a nuclear protein required for cell cycle progression in G1. Genes Dev 7: 812-821, 1993.

24. Masamha CP and Benbrook DM: Cyclin D1 degradation is sufficient to induce G1 cell cycle arrest despite constitutive expression of cyclin E2 in ovarian cancer cells. Cancer Res 69: 6565-6572, 2009.

25. Yang M, Zhong J, Zhao M, Wang J, Gu Y, Yuan X, Sang J and Huang C: Overexpression of nuclear apoptosis-inducing factor 1 altered the proteomic profile of human gastric cancer cell MKN45 and induced cell cycle arrest at G1/S phase. PLoS One 9: e100216, 2014 